http://dx.doi.org/10.5902/1984686X26398

\title{
Temas em altas habilidades/superdotação na perspectiva de gênero: um estudo de revisão sistemática
}

\section{Giftedness topics in gender perspective: a systematic review study}

\section{Temas en altas capacidades en la perspectiva de género: un estudio de revisión sistemática}

\section{* Mayra Berto Massuda}

Doutoranda da Universidade Federal de São Carlos, São Carlos, São Paulo, Brasil.

mayramassuda@gmail.com

\section{** Rosimeire Maria Orlando}

Professora da Universidade Federal de São Carlos, São Carlos, São Paulo, Brasil. meiremorlando@gmail.com

Recebido: 29 de março de 2017

Aprovado: 15 de dezembro de 2018

\section{RESUMO}

As pesquisas acerca das altas habilidades/superdotação (ah/sd) cresceram consideravelmente no cenário nacional após 1990 e mais vertiginosamente após 2008. Apesar disso, ainda são escassos os estudos que relacionam a temática das ah/sd e o gênero feminino. Assim, na tentativa de compreender os caminhos percorridos e os vales a serem desbravados nas pesquisas sobre esse tema, este estudo tem por objetivo analisar artigos pertinentes à temática, publicados em periódicos científicos entre os anos $2007 \mathrm{e}$ 2016, a fim de discutir principalmente a que objetivos eles atendem, quais foram os avanços alcançados e quais pontos têm sido negligenciados nesse contexto. O método adotado na pesquisa foi exploratório do tipo revisão sistemática de pesquisas empíricas publicadas em periódicos indexados em duas bases de dados: a Scientific Electronic Library Online (SciELO) e os Periódicos da Coordenação de Aperfeiçoamento de Pessoal de Nível Superior (CAPES). As buscas resultaram em 25 artigos que foram submetidos à técnica de revisão sistemática. Dentre os resultados levantados, destacam-se três grandes temáticas: análise das diferenças entre gêneros, estereótipos de gênero e aspirações profissionais. Nesse cenário, enfatiza-se a frequente utilização de escalas, scores e provas psicométricas na comparação entre as diferenças de gênero, a presença de estereótipos de gênero nos processos de identificação das ah/sd e também as diferenças de aspirações de carreira e de estilo de vida de homens e mulheres com ah/sd.

Palavras-chave: Superdotação; Gênero; Mulheres.

\section{ABSTRACT}

Research on giftedness have grown considerably in the Brazilian scientific scenario after 1990 and more vertiginously after 2008. Nevertheless, there is still little research about giftedness and the feminine gender. Thus, in an attempt to understand the limited literature and the challenges of this theme, this study aims to analyze articles on giftedness and 
gender published in scientific journals between 2007 and 2016 in order to discuss what propose they set, what progress they achieved and what points were neglected in this context. The method used in the research was the systematic review of empirical researches published in scientific journals indexed in two databases: Scientific Electronic Library Online (SciELO) and CAPES Scientific Journals Gateway. The investigation resulted in 25 articles that were analyzed using the systematic review technique. Among the results obtained, three main themes are highlighted: gender differences analysis, gender stereotypes and professional aspirations. We emphasize the frequent use of scales, scores and psychometric tests to compare gender differences, the presence of gender stereotypes in giftedness identification processes and also the differences in professional aspirations and lifestyle of gifted men and women.

Keywords: Giftedness; Gender; Women.

\section{RESUMEN}

Las investigaciones sobre las altas capacidades (AC) crecieron considerablemente en el escenario nacional después de 1990 y más vertiginosamente después de 2008. A pesar de ello, todavía son escasos los estudios que relacionan la temática de las ah / sd y el género femenino. Así, en el intento de comprender los caminos recorridos y los valles a ser desbravados en las investigaciones sobre ese tema, este estudio tiene por objetivo analizar artículos pertinentes a la temática, publicados en revistas científicas entre los años 2007 y 2016, a fin de discutir principalmente a qué objetivos atienden, cuáles fueron los avances alcanzados y qué puntos se han descuidado en ese contexto. El método utilizado en la investigación fue exploratorio del tipo revisión sistemática de investigaciones empíricas publicadas en revistas indexadas en dos bases de datos: la Scientific Electronic Library Online (SciELO) y los Periódicos da Coordenação de Aperfeiçoamento de Pessoal de Nível Superior (CAPES). Las búsquedas resultaron en 25 artículos que fueron sometidos a la técnica de revisión sistemática. Entre los resultados planteados, se destacan tres grandes temáticas: análisis de las diferencias entre géneros, estereotipos de género y aspiraciones profesionales. En este escenario, se enfatiza la frecuente utilización de escalas, puntuaciones y pruebas psicométricas en la comparación entre las diferencias de género, la presencia de estereotipos de género en los procesos de identificación de las ah / sd y también las diferencias de aspiraciones de carrera y de estilo de vida vida de hombres y mujeres con ah / sd.

Palabras clave: Altas capacidades; Género; Mujeres.

\section{Introdução}

Pessoas com potencial acima da média chamam a atenção de especialistas há milênios. A valorização do intelecto humano pelos atenienses e o reconhecimento do alto desempenho físico pelos espartanos são breves exemplos de como o alto nível de inteligência e performance geram um misto de adoração e curiosidade (GAMA, 2006). 
http://dx.doi.org/10.5902/1984686X26398

Atualmente, esse potencial é nomeado por diversos pesquisadores da Educação Especial como "altas habilidades", "superdotação", "talento" e "dotação", entre outras terminologias. Essa grande variedade de termos é um indicativo de autores que se aproximam ou divergem em suas defesas teóricas, embora todos estejam, de maneira geral, falando do alto nível de capacidade e desempenho.

Segundo Freitas (2014), as pesquisas relativas às altas habilidades/superdotação (ah/sd) ${ }^{1}$ têm ganhado espaço no cenário nacional, principalmente após a década de 1990, com o estabelecimento da Declaração Mundial sobre Educação para Todos (UNESCO, 1990), da Declaração de Salamanca (BRASIL, 1994) e, mais recentemente, da Política Nacional de Educação Especial na Perspectiva da Educação Inclusiva (BRASIL, 2008). Isso se torna notável se compararmos os estudos de Freitas e Pérez (2009), e o mais recente de Freitas (2014).

Se Freitas e Pérez (2009) apontam a existência de apenas sete teses de Doutorado e 50 dissertações de Mestrado com foco explícito na área de ah/sd até 2009, o estudo atualizado de Freitas (2014) já indica um aumento considerável entre 2009 e 2012, quando foram levantadas 15 teses de Doutorado e 96 dissertações de Mestrado. Apesar do aparente avanço, esse número representava apenas $4 \%$ dos estudos na área de Educação Especial na época.

Desse universo de pesquisas, menos ainda são as que abordam as ah/sd pela perspectiva do gênero ${ }^{2}$, fato que, diante da prevalência do gênero masculino em programas de atendimento às ah/sd e nas matrículas de aceleração (FERNANDES, 2015; PETERSON, 2013; PRADO; FLEITH; CARMO, 2011; MAIA-PINTO; FLEITH, 2004), ratifica a importância de pesquisas relativas à identificação e ao desenvolvimento de mulheres com ah/sd.

Nesse sentido, estudos como o de Ogeda, Pedro e Chacon (2016), confirmam a escassez de pesquisas sobre gênero dentro da temática. As pesquisadoras realizaram um levantamento na Scientific Library Online (SciELO) e na Biblioteca Digital Brasileira de Teses e Dissertações (BDTD), e conseguiram elencar apenas três artigos e uma

\footnotetext{
${ }^{1}$ Adotou-se, neste estudo, o termo "altas habilidades/superdotação" (ah/sd). Entretanto, nas citações diretas, foram respeitadas as terminologias originais utilizadas por cada autor.

${ }^{2}$ Em primeira instância, distingue-se aqui gênero de sexo. Segundo Scott (1995), enquanto sexo é um atributo biológico, gênero é como categoria de análise relacional, centrada na significação, no poder e no ator, constitutiva das relações sociais, em que estão presentes as influências histórica, social e política.
} 
http://dx.doi.org/10.5902/1984686X26398

dissertação que abordavam a questão das ah/sd e gênero, reforçando a hipótese de que há poucas produções nacionais na área.

Assim, este estudo parte dos seguintes questionamentos: Quais são as pesquisas científicas nacionais e internacionais sobre ah/sd na perspectiva de gênero? E quais as contribuições que essas pesquisas oferecem para o cenário científico atual?

Diante do interesse em conhecer a produção científica nacional e internacional acerca das ah/sd e o gênero, estabeleceu-se como objetivo: realizar a revisão sistemática de artigos pertinentes à temática, publicados em periódicos científicos entre os anos 2007 e 2016, a fim de discutir, principalmente, quais foram as principais temáticas abordadas nesses estudos, a que objetivos eles atendem, quais os avanços alcançados e quais pontos têm sido negligenciados nesse contexto.

\section{Método}

Utilizou-se o método exploratório a fim de iniciar o esclarecimento e a (re)elaboração de conceitos relativos ao tema pesquisado, formulando problemas e hipóteses mais precisos para estudos futuros (GIL, 2011). Como técnica, foi utilizada a revisão sistemática, com a intenção de reunir, avaliar criticamente e sintetizar os resultados de múltiplos estudos de maneira reflexiva, crítica e compreensiva, permitindo a exploração do tema de maneira consistente e sistematizada (COSTA; ZOLTOWSKI, 2014).

\section{Procedimentos}

Como procedimentos de pesquisa, foram respeitadas as 8 etapas básicas propostas por Costa e Zoltowski (2014) com base nos estudos de Akobeng (2005).

$\mathrm{Na}$ primeira etapa delimitou-se como escopo somente as pesquisas científicas que investigassem as ah/sd sob a perspectiva do gênero com o intuito de estabelecer uma relação clara entre os conceitos investigados e o panorama dos achados mais atuais sobre o tema.

A segunda etapa compreendeu a delimitação das fontes de dados, sendo escolhidas a SciELO, base nacional que abrange diversas áreas, e o Portal de Periódicos da CAPES, base de dados mais ampla, que agrega tanto periódicos nacionais quanto internacionais das mais diversas áreas e temas.

A terceira etapa contou com a eleição de palavras-chave para a busca, que foi realizada de forma a sintetizar conceitos investigados, buscando encontrar um número 
http://dx.doi.org/10.5902/1984686X26398

representativo de trabalhos por meio de uma string (conjunto de descritores com operadores booleanos) que unifica os procedimentos de busca. Foram utilizadas nas buscas as seguintes palavras-chave: "gifted", "talent", "female", "females", "gender", "women" e "woman", em inglês, com 10 possibilidades de string, provenientes das combinações das palavras dos dois campos semânticos diferentes; e "superdotação", "altas habilidades", "talento", "mulher", "gênero" e "feminino", com nove combinações de string.

A quarta etapa consistiu na busca padronizada e sistematizada dos dados, bem como em seu armazenamento. Foram obedecidos os seguintes critérios de seleção: a) ser artigo com resultados empíricos publicado em periódico; b) ter sido publicado entre 2007 e 2016; c) ter sido publicado em língua portuguesa, espanhola ou inglesa; d) apresentar no resumo e nas palavras-chave informações suficientes que especificassem se 0 artigo abordava 0 tema das ah/sd pela perspectiva do gênero; e) estar disponível para acesso de pesquisadores institucionais.

$\mathrm{Na}$ quinta etapa foi realizada a seleção de artigos pelo resumo, de acordo com critérios estabelecidos. Assim, na primeira análise dos arquivos, foi identificado se as publicações eram pertinentes à área e à proposta de estudo, excluindo aquelas que não fossem.

$\mathrm{Na}$ sexta etapa foi realizada a leitura detalhada e a extração dos dados mais significativos dos artigos selecionados, em que foram considerados: os objetivos, o método, os instrumentos e técnicas de pesquisa e os principais resultados dos estudos, já construindo uma planilha organizada para a análise dos mesmos.

A sétima etapa se concentrou na análise dos artigos para a discussão e comparação dos mesmos, agrupando-os por temática e subtemática, considerando, principalmente, quais foram os resultados alcançados e quais pontos têm sido negligenciados dentro desse contexto de pesquisa. Em seguida, na oitava etapa, foi realizada a síntese e interpretação dos dados, explorando as similaridades e diferenças das publicações levantadas.

\section{Resultados e Discussão}

No total, foram levantados 25 artigos empíricos relacionados às ah/sd na perspectiva de gênero publicados em português, inglês ou espanhol, entre 2007 e 2016, nas bases de dados da SciELO e do Portal de Periódicos da CAPES, sendo apenas dois artigos brasileiros.

De maneira geral, as publicações podem ser agrupadas em três grandes temáticas: 
http://dx.doi.org/10.5902/1984686X26398

- Análise das diferenças entre os gêneros - abordando diferenças cognitivas entre homens e mulheres com ah/sd, e diferenças em relação à superexcitabilidade e ao autoconceito;

- Estereótipos de gênero - abordando a influência dos estereótipos no processo de identificação de pessoas com ah/sd, e no processo de construção da identidade da mulher com ah/sd;

- Aspirações de carreira de pessoas com ah/sd - abordando a discussão sobre as aspirações de carreira de mulheres com ah/sd, seus fatores precedentes, sua relação com a parentalidade e percepções e prospecções de carreira em STEM (Science, Technology, Engineering and Mathematics) para pessoas com ah/sd.

O quadro 1 apresenta a organização dos artigos levantados a partir das três grandes temáticas e suas respectivas subtemáticas.

Quadro1 - Artigos empíricos sobre ah/sd e gênero publicados entre 2006 e 2017

\begin{tabular}{|c|c|c|c|}
\hline Temática & Subtemática & Título do Artigo & $\begin{array}{l}\text { Autor } \\
\text { (Ano) }\end{array}$ \\
\hline \multirow{8}{*}{$\begin{array}{c}\text { Análise das diferenças } \\
\text { entre os gêneros }\end{array}$} & \multirow{4}{*}{$\begin{array}{c}\text { Diferenças cognitivas } \\
\text { entre homens e } \\
\text { mulheres com ah/sd }\end{array}$} & $\begin{array}{l}\text { Gender differences in spatial } \\
\text { ability: relationship to } \\
\text { spatial experience among } \\
\text { chinese gifted students in } \\
\text { Hong Kong }\end{array}$ & $\begin{array}{l}\text { Chan } \\
(2007)\end{array}$ \\
\hline & & $\begin{array}{l}\text { Gender and other group } \\
\text { differences in performance } \\
\text { on Off-leval Tests: Changes } \\
\text { in the 21st century }\end{array}$ & $\begin{array}{l}\text { Olszewski- } \\
\text { Kubilius e Lee } \\
\text { (2011) }\end{array}$ \\
\hline & & $\begin{array}{l}\text { Diferencias em el desarollo } \\
\text { cognitivo y socioemocional } \\
\text { según sexo }\end{array}$ & $\begin{array}{l}\text { Mathiesen et al. } \\
\text { (2013) }\end{array}$ \\
\hline & & $\begin{array}{l}\text { Creatividad científica y alta } \\
\text { habilidad: diferencias de } \\
\text { género y nivel educativo }\end{array}$ & $\begin{array}{c}\text { Esparza Molina et } \\
\text { al. } \\
\text { (2015) }\end{array}$ \\
\hline & \multirow{3}{*}{$\begin{array}{l}\text { Diferenças em relação } \\
\text { à superexcitabilidade }\end{array}$} & $\begin{array}{c}\text { Gifted adolescents' } \\
\text { overexcitabilities and self- } \\
\text { concepts: an analysis of } \\
\text { gender and grade level }\end{array}$ & $\begin{array}{l}\text { Gross, Rinn e } \\
\text { Jamieson } \\
\text { (2007) }\end{array}$ \\
\hline & & $\begin{array}{c}\text { A comparison of } \\
\text { Dabrowski's } \\
\text { overexcitabilities by gender } \\
\text { for American and Korean } \\
\text { high school gifted students }\end{array}$ & $\begin{array}{c}\text { Piirto, } \\
\text { Montgomery e } \\
\text { May } \\
(2008)\end{array}$ \\
\hline & & $\begin{array}{l}\text { Gender identity and the } \\
\text { overexcitability profiles of } \\
\text { gifted college students }\end{array}$ & $\begin{array}{l}\text { Miller, Falk e } \\
\text { Huang } \\
\text { (2009) }\end{array}$ \\
\hline & $\begin{array}{l}\text { Diferenças em relação } \\
\text { ao autoconceito }\end{array}$ & $\begin{array}{l}\text { The role of gender and } \\
\text { previous participation in }\end{array}$ & $\begin{array}{l}\text { Cunningham e } \\
\text { Rinn } \\
\text { (2007) }\end{array}$ \\
\hline
\end{tabular}


http://dx.doi.org/10.5902/1984686X26398

\begin{tabular}{|c|c|c|c|}
\hline & & $\begin{array}{c}\text { a summer program on gifted } \\
\text { adolescents' self-concepts } \\
\text { over time }\end{array}$ & \\
\hline & & $\begin{array}{l}\text { Female 'big fish' swimming } \\
\text { against the tide: the 'big- } \\
\text { fish-little-pond effect' and } \\
\text { gender-ration in special } \\
\text { gifted classes }\end{array}$ & $\begin{array}{l}\text { Preckel et al. } \\
\text { (2008a) }\end{array}$ \\
\hline & & $\begin{array}{l}\text { Gender differences in gifted } \\
\text { and average-ability } \\
\text { students: comparing girls' } \\
\text { and boys' achievement, self- } \\
\text { concept, interest, and } \\
\text { motivation in Mathematics }\end{array}$ & $\begin{array}{l}\text { Preckel et al. } \\
\qquad(2008 b)\end{array}$ \\
\hline & & $\begin{array}{c}\text { Gender Differences in Gifted } \\
\text { Adolescents' Math/ Verbal } \\
\text { Self-Concepts and Math/ } \\
\text { Verbal Achievement: } \\
\text { Implicaitons for the STEM } \\
\text { fields }\end{array}$ & Rinn et al. (2008) \\
\hline & & $\begin{array}{l}\text { A canonical correlation } \\
\text { analysis of the influence of } \\
\text { social comparison, gender, } \\
\text { and grade level on the } \\
\text { multidimensional self- } \\
\text { concepts of gifted } \\
\text { adolescents }\end{array}$ & Rinn et al. (2009) \\
\hline & & $\begin{array}{c}\text { Grade and gender } \\
\text { differences in gifted } \\
\text { students' self-concepts }\end{array}$ & $\begin{array}{l}\text { Rudasill et al. } \\
\qquad(2009)\end{array}$ \\
\hline & & $\begin{array}{l}\text { The gifted rating scales- } \\
\text { school form: a validation } \\
\text { study based on ages, } \\
\text { gender, and race }\end{array}$ & $\begin{array}{l}\text { Pfeiffer, Petscher } \\
\text { e Kumtepe } \\
\text { (2008) }\end{array}$ \\
\hline & & $\begin{array}{l}\text { Gifted girls: gender bias in } \\
\text { gifted referrals }\end{array}$ & $\begin{array}{c}\text { Bianco et al. } \\
(2011)\end{array}$ \\
\hline & $\begin{array}{l}\text { estereótipos no } \\
\text { processo de } \\
\text { identificação de } \\
\text { pessoas com ah/sd }\end{array}$ & $\begin{array}{l}\text { Práticas pedagógicas } \\
\text { reprodutoras de } \\
\text { desigualdades: a } \\
\text { subrepresentação de } \\
\text { meninas entre alunos } \\
\text { superdotados }\end{array}$ & $\begin{array}{l}\text { Reis e Gomes } \\
\text { (2011) }\end{array}$ \\
\hline Estereótipos de gênero & & $\begin{array}{c}\text { Mathematically gifted } \\
\text { adolescent females' mixed } \\
\text { sentiment toward gender } \\
\text { stereotypes }\end{array}$ & $\begin{array}{c}\text { Kao } \\
(2015)\end{array}$ \\
\hline & $\begin{array}{l}\text { A influência dos } \\
\text { estereótipos no } \\
\text { processo de }\end{array}$ & $\begin{array}{l}\text { A case study of the suicide } \\
\quad \text { of a gifted female } \\
\text { adolescent: implications for } \\
\text { prediction and prevention }\end{array}$ & $\begin{array}{l}\text { Hyatt } \\
(2010)\end{array}$ \\
\hline & $\begin{array}{c}\text { construção da } \\
\text { identidade da mulher } \\
\text { com ah/sd }\end{array}$ & $\begin{array}{l}\text { The Dilemmas of Peer } \\
\text { Relationship confronting } \\
\text { mathematically gifted } \\
\text { female adolescents: nine } \\
\text { cases in Taiwan }\end{array}$ & $\begin{array}{c}\text { Kao } \\
(2011)\end{array}$ \\
\hline
\end{tabular}


http://dx.doi.org/10.5902/1984686X26398

\begin{tabular}{|c|c|c|c|}
\hline & & $\begin{array}{c}\text { A mulher com altas } \\
\text { habilidades/ superdotação à } \\
\text { procura de uma identidade }\end{array}$ & $\begin{array}{l}\text { Pérez e Freitas } \\
\text { (2012) }\end{array}$ \\
\hline \multirow{5}{*}{$\begin{array}{l}\text { Aspirações de carreira } \\
\text { de pessoas com ah/sd }\end{array}$} & $\begin{array}{c}\text { Fatores precedentes à } \\
\text { escolha profissional }\end{array}$ & $\begin{array}{c}\text { Gender Differences in gifted } \\
\text { students' advice on solving } \\
\text { the world's problems }\end{array}$ & $\begin{array}{l}\text { Malin e Makel } \\
\qquad(2012)\end{array}$ \\
\hline & \multirow[t]{2}{*}{ Aspirações de carreira } & $\begin{array}{l}\text { Gifted american and german } \\
\text { adolescent women: a } \\
\text { longitudinal examination of } \\
\text { attachment, separation, } \\
\text { gender roles, and career } \\
\text { aspirations }\end{array}$ & $\begin{array}{l}\text { Fiebig } \\
(2008)\end{array}$ \\
\hline & & $\begin{array}{l}\text { Longitudinal Change and } \\
\text { maternal influence on } \\
\text { Occupational Aspirations of } \\
\text { gifted female american and } \\
\text { german adolescents }\end{array}$ & $\begin{array}{c}\text { Fiebig e } \\
\text { Beauregard } \\
(2010)\end{array}$ \\
\hline & $\begin{array}{c}\text { Relação entre } \\
\text { aspiração de carreira e } \\
\text { parentalidade }\end{array}$ & $\begin{array}{l}\text { Work preferences, life } \\
\text { values, and personal views } \\
\text { of top math/science } \\
\text { gradutate students and the } \\
\text { profoundly gifted: } \\
\text { developmental changes and } \\
\text { gender differences during } \\
\text { emerging adulthood and } \\
\text { parenthood }\end{array}$ & $\begin{array}{l}\text { Ferriman, } \\
\text { Lubinski e } \\
\text { Benbow } \\
(2009)\end{array}$ \\
\hline & $\begin{array}{c}\text { Percepções e } \\
\text { prospecções de } \\
\text { carreira em STEM }\end{array}$ & $\begin{array}{l}\text { The STEM Pathway for } \\
\text { women: what has changed }\end{array}$ & $\begin{array}{l}\text { Heilbronner } \\
\text { (2013) }\end{array}$ \\
\hline
\end{tabular}

Fonte: Elaborado pelas autoras

Os estudos levantados que realizam análises das diferenças entre gênero foram divididos em três subtemáticas: diferenças cognitivas, superexcitabilidade e autoconceito.

Já há muito se discute diferenças cognitivas entre homens e mulheres. Nesse sentido, os estudos de Chan (2007), Olszewski-Kubilius e Lee (2011), Mathiesen et al. (2013), Esparza Molina et al. (2015) discutem a diferença de habilidades e de desenvolvimento cognitivo de meninos e meninas com ah/sd.

A pesquisa desenvolvida por Chan (2007) avaliou as habilidades espaciais de alunos/as com ah/sd de Hong Kong e mostrou pequenas diferenças de gênero nas experiências com artes visuais, favorecendo as meninas; e nas experiências com orientação visual, favorecendo meninos. $O$ autor destaca que a experiência espacial teve um efeito mais marcante em meninas e sugere que encorajá-las a participar de experiências espaciais pode reduzir a discrepância de habilidades espaciais entre os gêneros.

Olszewski-Kubilius e Lee (2011) avaliaram as diferenças de gênero nos scores de alunos/as com ah/sd da Northwestern University's Midwest Academic Talent Search 
http://dx.doi.org/10.5902/1984686X26398

(Estados Unidos da América) em subtestes de provas como o Scholastic Aptitude Test (SAT), o American College Test (ACT) e o EXPLORE, ao longo de 8 anos. Os autores indicam que os meninos apresentaram scores mais altos nos subtestes de matemática e ciências, enquanto as meninas apresentaram scores mais altos nos testes verbais, sendo que essas diferenças permaneceram estáveis e com efeitos de tamanhos menores ou insignificantes após o período de investigação.

Em estudo similar, porém com metodologia e instrumentos diferentes, Mathiesen et al. (2013) investigaram as diferenças de gênero de alunos/as com ah/sd participantes do programa Talentos UdeC, no Chile. Não foram encontradas diferenças relevantes no desenvolvimento cognitivo em matemática e linguagem entre os dois grupos. Entretanto, quanto ao desenvolvimento socioemocional, observou-se uma vantagem feminina em todos os indicadores, o que revela que elas são mais adaptadas social e emocionalmente, têm melhor conduta pró-social, maior motivação acadêmica e responsabilidade social.

Com outra perspectiva, o estudo de Esparza Molina et al. (2015) investigou as diferenças de gênero e de nível educativo em relação à criatividade científica de alunos/as com ah/sd da Espanha. Os resultados das provas indicaram que os meninos tiveram melhores rendimentos em duas tarefas (as que exigiam capacidade de gerar diferentes hipóteses sobre problemas de diferentes áreas e as que exigiam maior domínio das ciências), ao passo que as meninas apresentaram melhor rendimento também em duas tarefas (as que exigem a avaliação de hipóteses e as que exigem avalição de evidências nas áreas de física e ecologia). Todavia, os autores concluem que, em geral, os meninos apresentaram rendimento superior às meninas em relação à criatividade científica.

Esses estudos apontam uma característica frequente nas pesquisas sobre ah/sd e gênero: a tentativa de apontar e justificar diferenças cognitivas e de habilidades entre os gêneros por meio de comparações realizadas por escalas, scores e provas psicométricas. Assim, é necessário destacar que embora essas provas e instrumentos tenham grande validade - científica ou acadêmica - foram construídos a partir de uma perspectiva humana, carregada de visão ideológica e, por isso, não estão livres de vieses culturais e sociais (PATTO, 1997).

Outro tema frequente nas pesquisas de comparação entre gêneros é a superexcitabilidade, como os estudos de Gross, Rinn e Jamieson (2007), Piirto, Montgomery e May (2008), Miller, Falk e Huang (2009). 
http://dx.doi.org/10.5902/1984686X26398

Isto posto, o estudo de Gross, Rinn e Jamieson (2007), realizado nos Estados Unidos, analisou a relação entre a superexcitabilidade e 0 autoconceito ${ }^{3}$ de adolescentes com ah/sd, avaliando também os efeitos do gênero e do nível educativo. Os resultados mostraram que as meninas obtiveram níveis mais altos de superexcitabilidade sensorial, imaginativa e emocional, o que pode influenciar negativamente o autoconceito dessas meninas, levando a uma visão negativa de si mesma e dificultando ou impedindo o desenvolvimento de seu potencial.

Em estudo parecido, Piirto, Montgomery e May (2008) abordaram a diferença entre gêneros na superexcitabilidade emocional, intelectual, imaginativa, sensorial e psicomotora de alunos/as americanos e sul-coreanos. A pesquisa se baseou principalmente na comparação quanto à origem dos participantes e concluiu que as diferenças culturais entre as duas populações podem influenciar a superexcitabilidade tanto em meninos quanto em meninas.

O estudo de Miller, Falk e Huang (2009), realizado com alunos de uma universidade dos Estados Unidos que tivessem participado anteriormente de um programa de atendimento às ah/sd mostrou que, em geral, há uma relação maior entre o gênero e a superexcitabilidade, do que entre o sexo e a superexcitabilidade. Esse resultado reflete a influência dos papeis tradicionais de gênero nos scores de superexcitabilidade sensorial, emocional e intelectual dos participantes e coloca em pauta a necessidade de discussão sobre gênero e sexo. Os autores sugerem ainda que, se os papéis sociais não fossem definidos pelo gênero, os traços de personalidade poderiam ser valorizados mais apropriadamente. Assim, se a exigência dos professores e pais fossem menos tendenciosas em relação a atribuições de papeis sociais de acordo com o gênero, tanto meninas quanto meninos seriam encorajados a responder independentemente das normas tradicionais de gênero, confiando na singularidade de sua identidade.

Dessa forma, nota-se que o gênero - visto como resultado das relações sociais exerce grande influência na avaliação psicométrica da superexcitabilidade. Entretanto, é necessário considerar que esse não é o único fator a intervir nas diferenças dos scores de superexcitabilidade, sendo importante destacar os fatores demográficos e também a

\footnotetext{
${ }^{3}$ Segundo Byrne, autoconceito é o conjunto de "atitudes, sentimentos e conhecimentos sobre nossa própria capacidade, habilidade, aparência e aceitação social” (BYRNE,1984, p. 429, tradução nossa).
} 
http://dx.doi.org/10.5902/1984686X26398

influência dos estereótipos de gênero veiculados principalmente pelos professores no ambiente escolar.

Ademais, acredita-se também que o meio social influencia sobremaneira não só o nível de superexcitabilidade, mas também os níveis de autoconceito de homens e mulheres com ah/sd. É o que discutem os estudos de Cunningham e Rinn (2007), Preckel et al. (2008a, 2008b), Rinn et al. (2008, 2009), e Rudasill et al. (2009).

Cunningham e Rinn (2007) retomam estudos anteriores e apontam a dificuldade de medir, analisar e discutir a comparação de autoconceito entre os gêneros, diante de diferenças metodológicas entre as pesquisas. De forma geral, os autores não chegaram a conclusões consistentes sobre as diferenças entre os gêneros no autoconceito ao investigar o engajamento de meninos e meninas em programas de verão dos Estados Unidos voltados para as ah/sd e a influência dessa participação no autoconceito geral e na estabilidade emocional. Entretanto, em pesquisa similar, avaliando a participação de alunos/as com ah/sd em programas de verão de enriquecimento escolar do mesmo país, Rudasill et al. (2009) apontam diferenças de gênero em inúmeros domínios de autoconceito, em que os scores das meninas, principalmente as mais velhas, apresentaram-se mais baixos que os dos meninos.

Uma das variáveis frequentes nas pesquisas sobre autoconceito é a relação gênero e matemática. Nesse sentido, os estudos de Preckel et al. (2008a, 2008b), realizados em Israel e na Alemanha, indicam que meninas com ah/sd apresentam níveis mais baixos de autoconceito acadêmico, de autoconceito em matemática e de interesse em matemática, bem como menor engajamento em desenvolver habilidades matemáticas e em atingir uma determinada performance. Além disso, Preckel et al. (2008a) afirmam que um número desproporcional de meninos e meninas em classes de alunos com ah/sd têm um efeito negativo no autoconceito acadêmico de meninas, enquanto não exerce nenhuma influência no autoconceito acadêmico de meninos.

A diferença de gênero também chama a atenção em relação à habilidade e ao autoconceito verbal. Assim, os estudos de Rinn et al. (2008, 2009), realizados nos Estados Unidos concluem que as alunas com ah/sd apresentam tanto habilidades verbais quanto autoconceito verbal mais altos que os dos alunos com ah/sd. O estudo de Rinn et al. (2009) ainda corrobora a influência do fator gênero, nível educativo e comparação social nas variáveis de autoconceito de habilidade física, de estabilidade emocional, verbal e de 
http://dx.doi.org/10.5902/1984686X26398

interações parentais. Nessas, as meninas pareceram mais suscetíveis a baixo autoconceito, principalmente quando este estava submetido à comparação social.

Destaca-se nessas pesquisas a frequente comparação de scores de autoconceito principalmente nos domínios verbal, matemático e de estabilidade emocional, sendo possível notar que essas variáveis são frequentes nessa área de pesquisa. Os estudos revelam a diferença de gênero em relação ao autoconceito e a influência que isso pode exercer na carreira e no desenvolvimento do potencial dessas meninas. Os resultados também indicam a importância de esforços para criar ambientes em que meninas consigam ver a si mesmas como agentes positivos em suas próprias vidas e avaliem a si mesmas positivamente (RUDASILL et al., 2009).

De maneira geral, é possível concluir que os estudos centrados na temática das diferenças de gênero entre pessoas com ah/sd são resultado de investigações psicométricas, comprometidas em avaliar diferenças cognitivas, de superexcitabilidade e de autoconceito de homens e mulheres com ah/sd. Fatores sociais como a origem demográfica, a idade, o acesso a programas específicos para o atendimento das ah/sd e a persistência de estereótipos de gênero revelaram-se importantes influenciadores dessas diferenças.

O segundo grupo de publicações levantadas na pesquisa refere-se aos estereótipos de gênero, não só em relação à identificação de alunos/as com ah/sd, em que se enquadram os estudos de Pfeiffer, Petscher e Kumtepe (2008), Bianco et al. (2011), Reis e Gomes (2011) e Kao (2015), como também em relação à construção da identidade como mulher com ah/sd, em que se enquadram os estudos de Hyatt (2010), Kao (2011), Pérez e Freitas (2012).

Nesse sentido, Bianco et al. (2011) apontam que as indicações de alunos com ah/sd por parte dos professores do Colorado e da Flórida (Estados Unidos) são influenciadas pelo gênero, sendo que os professores são mais propensos a indicar alunos meninos do que alunas meninas com as mesmas características. No estudo, as descrições do aluno elaboradas pelos professores, bem como as razões para sua indicação, diferem consideravelmente de acordo com o gênero do aluno, o que ilustra a existência do preconceito de gênero na percepção, expectativas e crenças dos professores.

Em concordância com essa pesquisa, Reis e Gomes (2011) apontam a subestimativa do potencial feminino na seleção e na indicação de alunos/as para um programa brasileiro de atendimento às ah/sd. O estudo mostra que, principalmente a indicação realizada por 
http://dx.doi.org/10.5902/1984686X26398

professores do ensino regular está enviesada por estereótipos de gênero, o que revela a necessidade de formação adequada para que os professores sejam capazes de realizar as indicações com equidade.

Desse modo, é necessário retomar o estudo de Pfeiffer, Petscher e Kumtepe (2008), que investigou a validade das Gifted Rating Scales (GRS), instrumento de identificação de alunos com ah/sd, desenvolvido por pesquisadores da Duke University (Estados Unidos). Os autores exploraram os efeitos do gênero, raça/etnia, idade e familiaridade dos avaliadores e concluíram que há uma alta consistência interna para as seis escalas do instrumento e que não há efeitos de diferenças de gênero na identificação de estudantes.

Com isso, é possível dizer que o processo de identificação das ah/sd é um momento decisivo para o reconhecimento e posterior desenvolvimento do potencial de alunas com ah/sd, mas que, entretanto, está marcado pela frequente presença de vieses culturais e de estereótipos de gênero, que deveriam ser estudados e discutidos na busca de práticas educacionais mais equânimes.

Considerando que os estereótipos de gênero exercem determinante influência no processo de identificação de alunos/as com ah/sd, Kao (2015) aponta que eles aparecem também nas falas das próprias alunas com ah/sd. Ao analisar os estereótipos de gênero presentes nos discursos de nove meninas taiwanesas com ah/sd na área de matemática, Kao (2015) conclui que elas apresentam sentimentos ambivalentes em relação aos estereótipos de gênero. Por um lado, elas afirmam que se sentem desconfortáveis e até mesmo indignadas com a desigualdade de gênero, reprovam estereótipos que preconizam a competência inferior da mulher, negam a importância de beleza e expressam grandes objetivos para o futuro, baseados, principalmente em aspirações de carreiras bemsucedidas. Por outro lado, concordam com o estereótipo de que as mulheres teriam uma tendência a formar grupos de amizade fechados e teriam mente mais fechada. Além disso, muitas se identificam com características e interesses considerados masculinos, preferem ter amizades com meninos e buscam participar mais de atividades com eles do que com meninas, muitas vezes se recusando a participar de atividades que envolvam exclusivamente meninas. Kao (2015) destaca que meninas com ah/sd geralmente possuem uma alta consciência e sensibilidade, o que muitas vezes pode fazer com que sua ambivalência em relação aos estereótipos de gênero resulte em conflito interno e estresse excessivo. 
http://dx.doi.org/10.5902/1984686X26398

As diferenças cognitivas e psicológicas entre homens e mulheres com ah/sd, bem como os estereótipos de gênero que permeiam todos os processos de identificação das ah/sd exercem papel importante na construção da identidade da mulher com ah/sd. Nesse sentido, o estudo de Hyatt (2010) pode ser considerado um alerta de urgência para a discussão desses aspectos.

Hyatt (2010) partiu de entrevistas, registros e materiais videogravados de uma adolescente com ah/sd dos Estados Unidos que se suicidou aos 18 anos. A autora discutiu as variáveis pessoais, ambientais e culturais que podem ter contribuído para o suicídio e apontou que a frustração, a raiva e a infelicidade da adolescente estavam relacionadas a sua experiência com o bullying, rejeição e incompreensão por pares, professores e gestores escolares. Os resultados indicam a necessidade de mudanças na educação para crianças e adolescentes com ah/sd e destacam a importância de dar a pais, professores e gestores mais informações aprofundadas acerca das características das ah/sd e dos indicadores de pensamentos suicidas, para que esses sejam prevenidos.

O estudo de Kao (2011) vai ao encontro desse alerta. Ao investigar as relações entre pares de adolescentes taiwanesas com ah/sd, Kao (2011) conclui que é necessário atentar para 6 temas que emergiram nos resultados: 1) a propensão à solidão; 2) a indiferença à popularidade; 3) o melhor relacionamento com pessoas com ah/sd; 4) a preferência pelo autodidatismo; 5) a preferência por salas com maioria masculina; e 6) maior apego à família do que a amigos. Segundo o autor, a inconsistência dos achados em relação à literatura da área, principalmente quanto à preferência por salas com maioria masculina, aponta a necessidade de olhar as ah/sd feminina por novos ângulos.

O estudo longitudinal de Pérez e Freitas (2012), realizado no Brasil, revisita a história de duas mulheres (47 e 50 anos) identificadas com ah/sd. Os resultados apontaram que, apesar das barreiras internas e externas vivenciadas por mulheres com ah/sd, as participantes tiveram uma progressiva aceitação dos indicadores e o reconhecimento das características de ah/sd após a sua identificação formal. As autoras ainda destacam a importância da identificação, já que o não reconhecimento ou o sub-reconhecimento de indicadores de ah/sd em mulheres pode levar à não-aceitação e à ocultação dessas características. Além disso, as autoras afirmam que o processo de construção positivo da identidade de mulher com ah/sd só foi possível porque estava alicerçado na troca com pares com ah/sd e na constante discussão sobre o tema. 
http://dx.doi.org/10.5902/1984686X26398

Desse modo, os estudos centrados nos estereótipos de gênero revelam diversas consequências da pressão social para que as meninas e mulheres com ah/sd correspondam a papéis sociais socialmente demarcados, começando pela subindicação e sub-representação nos programas de atendimento específico às ah/sd e culminando em sentimentos de solidão, rejeição e incompreensão por parte dos outros, que podem desencadear o sofrimento com o bullying e, em um caso específico, o suicídio. Destaca-se que a identificação formal tem grande importância para o auto reconhecimento das características das ah/sd para a aceitação desses indicadores.

Por fim, as aspirações de carreira de pessoas com ah/sd revelaram-se fortemente influenciadas pelo fator gênero, como apontam os estudos de Fiebig (2008), Ferriman, Lubinski e Benbow (2009), Fiebig e Beauregard (2010), Malin e Makel (2012) e Heilbronner (2013).

Malin e Makel (2012) abordam fatores precedentes à preferência de estilo de vida na idade adulta e à escolha de carreira profissional ao analisar textos elaborados por alunos/as com $\mathrm{ah} / \mathrm{sd}$. Os textos versavam sobre uma hipotética candidatura ao gabinete da presidência dos Estados Unidos para resolver problemas nacionais de grande importância. O estudo apontou diferenças significativas em relação ao tipo de problema selecionado por meninos e por meninas para discursarem. As escolhas de função e ocupação dentro do gabinete revelam valores dos participantes que podem exercer uma importante influência no futuro educacional e nas aspirações profissionais desses alunos.

Nesse mesmo sentido, os estudos de Fiebig (2008), Fiebig e Beauregard (2010) versam sobre as aspirações profissionais de adolescentes norte-americanas e alemãs com ah/sd. Fiebig (2008) aponta que ao longo de 4 anos, as participantes apresentaram mudanças em suas aspirações profissionais, sendo que seus desejos de conquistar papéis de liderança ou expertise na futura carreira diminuíram. Elas mostraram-se mais independentes em relação à interação materna e mais conservadoras em relação aos direitos e responsabilidades das mulheres, o que pode influenciar diretamente suas aspirações de carreira e a forma como correspondem à família e aos relacionamentos. Entretanto, o estudo de Fiebig e Beauregard (2011), realizado com técnicas e instrumentos diferentes, aponta que, estatisticamente, tanto as adolescentes americanas quanto as alemãs permaneceram consistentes em seus interesses profissionais com o passar de 4 anos. 
http://dx.doi.org/10.5902/1984686X26398

Ferriman, Lubinski e Benbow (2009) apontam a relação entre a parentalidade e a aspiração de carreira e preferência de estilo de vida de pessoas com ah/sd durante o início da vida adulta. As autoras apontam que embora homens e mulheres com ah/sd tenham muitas similaridades psicológicas, tomam estratégias diferentes para gerenciar múltiplos papéis na vida durante a década seguinte a sua educação formal. Por volta dos 30 anos, os homens com ah/sd tendem a focar e valorizar mais o status em sua carreira, já as mulheres com ah/sd despendem mais atenção em papéis relacionados à família, esposo, amigos e bem-estar social fora do trabalho. Os resultados ainda mostram que muitas mulheres sem filhos são mais centradas no trabalho do que aquelas que têm filhos.

Por fim, é importante destacar que o estudo de Heilbronner (2013) aborda fatores relacionados às diferenças dos padrões acadêmicos de homens e mulheres, bem como sua representação nos cargos na área de Ciências, Tecnologia, Engenharia e Matemática (STEM). A autora explorou as percepções de 360 finalistas e semifinalistas da competição Science Talent Search e concluiu não só que as mulheres se lembram de ter menor auto eficácia nas áreas de STEM na faculdade do que os homens, mas também que poucas escolheram cursos da área de STEM como majors. Proporcionalmente, mais mulheres do que homens entram na área de biologia e menos mulheres do que homens seguem áreas como engenharia ou física/astronomia. Uma grande proporção de mulheres mais velhas mencionou abandonar a área devido à falta de horários flexíveis e à necessidade de dedicar-se às responsabilidades familiares. Dessa forma, a autora aponta que é importante que as habilidades e interesses de mulheres jovens com ah/sd sejam estimuladas e desafiadas e que elas tenham acesso a oportunidades dentro da área de STEM.

Assim, na última temática abordada, as aspirações de carreiras de pessoas com ah/sd, é possível notar que o fator gênero exerce influência tanto nas escolhas profissionais quanto no estilo de vida de mulheres com ah/sd, principalmente dentro da área de STEM.

\section{Considerações Finais}

A revisão sistemática de publicações relativas às ah/sd dentro da perspectiva de gênero mostrou-se fundamental para compreender os avanços científicos nacionais e internacionais na área das ah/sd, além de questionar frequentes vieses encontrados nesses estudos. De modo geral, destacam-se três grandes temáticas investigadas: diferenças entre gêneros, estereótipos de gênero e aspirações profissionais. 
http://dx.doi.org/10.5902/1984686X26398

É frequente a utilização de escalas, scores e provas psicométricas para a avaliação de diferenças cognitivas e de habilidades entre homens e mulheres com ah/sd, bem como na análise dos níveis de superexcitabilidade e autoconceito. As diferenças levantadas por esses instrumentos apontam ora o destaque feminino nas áreas verbal, de linguagem, de artes visuais e de desenvolvimento socioemocional, ora o destaque masculino nas áreas de ciências, matemática e criatividade científica. Além disso, os estudos destacam que as mulheres com ah/sd estão mais sujeitas às consequências negativas resultantes dos altos níveis de superexcitabilidade e do baixo nível de autoconceito.

Os estereótipos de gênero mostraram-se marcantes tanto no processo de identificação das ah/sd, quanto na fala das próprias mulheres com ah/sd, o que reforça a necessidade de discussão da perspectiva de gênero nos processos de identificação das ah/sd, com práticas que visem a equidade e proporcionem o reconhecimento do potencial humano independente do gênero a que ele está agregado.

Os discursos que repetidamente reforçam as diferenças cognitivas entre os gêneros, somados aos estereótipos que fazem parte do imaginário tanto de professores, quanto das próprias meninas com ah/sd, resultam em diferenças significativas de aspirações profissionais e estilo de vida, frequentemente marcadas pela baixa expectativa, pela busca de carreiras ditas adequadas para o gênero feminino e, muitas vezes, pela dificuldade de gerenciamento de múltiplos papéis nas esferas familiares, sociais e profissionais.

Ao conduzir o estudo, foram encontradas limitações relativas à abrangência da busca realizada. Futuramente, em pesquisas mais extensas, os resultados podem ser ampliados se forem adotados outros critérios de seleção de artigos, como os descritores utilizados, as línguas de publicação, as bases de dados onde estão indexados, ano de publicação e disponibilidade de acesso aberto.

Ademais, alguns estudos alertam para a importância de um modelo teórico e de um processo de identificação que reduzam as desigualdades de gênero e, associada a isso, a disseminação de informações aprofundadas sobre ah/sd a professores e responsáveis. Destaca-se, por fim, a importância de discutir a temática também diferenciando sexo e gênero e de investir em práticas que proporcionem a equidade de gênero na identificação e no desenvolvimento das ah/sd. 


\section{Referências}

AKOBENG, A. K. Understanding systematic reviews and meta-analysis. Archives of Disease in Childhood, v. 90, p. 845-848, 2005.

BIANCO, M. et al. Gifted girls: gender bias in gifted referrals. Roeper review, v. 33, n.3, p. 170-181, 2011.

BRASIL. Declaração de Salamanca. Sobre Princípios, Políticas e Práticas na Área das Necessidades Educativas Especiais. Brasília: UNESCO, 1994. Disponível em: $<$ http://portal.mec.gov.br/seesp/arquivos/pdf/salamanca.pdf>. Acesso em: 04 abr. 2014.

MEC/SEESP. Políticas Nacionais de Educação Especial na Perspectiva da Educação Inclusiva, 2008. Disponível em: <http://portal.mec.gov.br/seesp/arqui vos/pdf/politica.pdf>. Acesso em: 03 abr. 2014.

BYRNE, B. M. The general/academic self-concept nomological network: a review of construct validation research. Review of Educational Research, v. 54, p. 427-456, 1984.

CHAN, D. W. Gender differences in spatial ability: relationship to spatial experience among chinese gifted students in Hong Kong. Roeper Review, v. 29, n. 4, p. 267-325, 2007.

COSTA, A. B.; ZOLTOWSKI, A. P. Como escrever um artigo de revisão sistemática. In: KOLLER, S. H.; COUTO, M. C. P.; HOHENDORF, J. Manual de Produção Científica. Porto Alegre: Penso, 2014. p. 55-70.

CUNNINGHAM, L. G.; RINN, A. N. The role of gender and previous participation in a summer program on gifted adolescents' self-concepts over time. Journal for the Education of the Gifted, v. 30, n. 3, p. 326-353, 2007.

ESPARZA MOLINA, F. J. et al. Creatividad científica y alta habilidade: diferencias de género y nível educativo. Aula, v. 21, p. 49-62, 2015.

FERRIMAN, K.; LUBINSKI, D.; BENBOW, C. P. Work preferences, life values, and personal views of top math/science graduate students and the profoundly gifted: developmental changes and gender differences during emerging adulthood and parenthood. Journal of Personality and Social Psychology, v. 97, n. 3, p. 517-532, 2009.

FERNANDES, H. S. Mensagem às famílias: a aceleração como estratégia educativa diferenciada. In: X Encontro Internacional de Educadores do CEDET-ASPAT, 2015, Poços de Caldas. Manual do participante. Poços de Caldas: ASPAT, 2015.

FIEBIG, J. N. Gifted american and german adolescent women: a longitudinal examination of attachment, separation, gender roles, and career aspirations. High Ability Studies, v. 19, n. 1, p. 67-81, 2008. 
FIEBIG, J. N.; BEAUREGARD, E. Longitudinal Change and Maternal Influence on Occupational Aspirations of Gifted Female American and German Adolescents. Journal for the Education of the Gifted, v. 34, n. 1, p. 45-67, 2010.

FREITAS, S. N. Ah/sd em pesquisa: um olhar dirigido. In: OMOTE, S.; OLIVEIRA, A. A. S.; CHACON, M. M. Ciência e Conhecimento em Educação Especial. São Carlos: Marquezini \& Manzini; ABPEE, 2014. p. 125-133.

FREITAS, S. N.; PÉREZ, S. G. P. B. Estado do conhecimento na área de Ah/sd no Brasil: uma análise das últimas décadas. In: ASSOCIAÇÃO NACIONAL DE PÓSGRADUAÇÃO EM PESQUISA E EDUCAÇÃO, 32., Caxambu, 2009. Anais... Caxambu: ANPED, 2009.

GAMA, M. C. S. S. Educação de Superdotados: teoria e prática. São Paulo: EPU, 2006.

GIL, A. C. Métodos e técnicas em pesquisa social. 6. ed. São Paulo: Atlas, 2011.

GROSS, C. M.; RINN, A. N.; JAMIESON, K. M. Gifted adolescents' overexcitabilities and self-concepts: an analysis of gender and grade level. Roeper Review, v. 29, n. 4, p. 240-248, 2007.

HEILBRONNER, N. N. The STEM Pathway for women: what has changed? Gifted Child Quarterly, v. 57, n. 1, p. 39-55, 2013.

HYATT, L. A case study of the suicide of a gifted female adolescent: implications for prediction and prevention. Journal for the Education of the Gifted, v. 33, n. 4., p.514535, 2010.

$\mathrm{KAO}, \mathrm{C}-\mathrm{Y}$. Mathematically gifted adolescent females' mixed sentiment toward gender stereotypes. Social Psychology Education, v. 18, n. 1, p. 17-55, 2015.

The dilemmas of peer relationships confronting mathematically gifted female adolescents: nine cases in Taiwan. Gifted Child Quarterly, v. 55, n. 2, p. 83-94, 2011.

MAIA-PINTO, R. R.; FLEITH, D. S. Avaliação das práticas educacionais de um programa de atendimento a alunos superdotados e talentosos. Psicologia Escolar e Educacional, v. 8, n. 1, p. 55-66, 2004.

MALIN, J.; MAKEL, M. C. Gender differences in gifted students' advice on solving the world's problems. Journal for the Education of the Gifted, v. 35, n. 2, p. 175-187, 2012.

MATHIESEN, M. E. et al. Diferencias en el desarollo cognitive y socioemocional según sexo. Estudios Pedagógicos, v. 39, n. 2, p. 199-2011, 2013.

MILLER, N. B.; FALK, R. F.; HUANG, Y. Gender identity and the overexcitability profiles of gifted college students. Roeper Review, v. 31, n. 3, p. 161-169, 2009. 
OGEDA, C. M. M.; PEDRO, K. M.; CHACON, M. C. M. A sub-representação da mulher no universo da superdotação. In: CONGRESSO INTERNACIONAL DE EDUCAÇÃO ESPECIAL E INCLUSIVA, 1.; JORNADA DE EDUCAÇÃO ESPECIAL, 13., Marília, 2016. Anais... Marília: UNESP, 2016.

OLSZEWSKI-KUBILIUS, P.; LEE, S-Y. Gender and other group differences in performance on off-level tests: changes in the $21^{\text {st }}$ century. Gifted Child Quarterly, v. 55, n. 1, p. 54-73, 2011.

PATTO, M. H. S. Para uma crítica da razão psicométrica. Psicologia USP, v. 8, n. 1, 1997.

PÉREZ, S. G. P. B.; FREITAS, S. N. A mulher com altas habilidades/superdotação: à procura de uma identidade. Revista Brasileira de Educação Especial, v. 18, n. 4, p.677-694, 2012.

PETERSON, J. Gender differences in identification of gifted youth and in gifted program participation: a meta-analysis. Contemporary Educational Psychology, v. 38, n. 4, p. 342-348, 2013.

PFEIFFER, S. I.; PETSCHER, Y.; KUMTEPE, A. The gifted rating scales-school form: a validation study based on age, gender and race. Roeper Review, v. 30, p. 140-146, 2008.

PIECHOWSKI, M. M. Overexcitabilities. In: RUNCO, M.; PRITZER, S. Encyclopedia of creativity. San Diego: Academic Press, 1999. p. 325-334.

PIIRTO, J.; MONTGOMERY, D.; MAY, J. A comparison of Dabrowski's overexcitabilities bu gender for American and Korean high school gifted students. High Ability Studies, v. 19, n. 2, p. 141-153, 2008.

PRADO, R. M.; FLEITH, D. S; CARMO, F. O Desenvolvimento do talento em uma perspectiva feminina. Psicologia: Ciência e Profissão, v. 31, n. 1, p. 134-145, 2011.

PRECKEL, F. et al. Female 'big fish' swimming against the tide: the 'big-fish-little-pond effect' and gender-ratio in special gifted classes. Contemporary Educational Psychology, v. 33, n. 1, p. 78-96, 2008a.

Gender differences in gifted and average-ability students: comparing girls' and boys' achievement, self-concept, interest, and motivation in Mathematics. Gifted Child Quarterly, v. 52, n. 2, p. 146-159, 2008b.

REIS, A. P. P. Z.; GOMES, C. A. Práticas pedagógicas reprodutoras de desigualdades: a sub-representação de meninas entre alunos superdotados. Estudos Feministas, v. 19, n. 2, p. 503-519, 2011. 
RINN, A. et al. A canonical correlation analysis of the influence of social comparison, gender, and grade level on the multidimensional self-concepts of gifted adolescents. Social Psychology Education, v. 12, n. 2, p. 251-269, 2009.

Gender differences in gifted adolescents' math/verbal self-concepts and math/verbal achievement: implications for the STEM Fields. Journal for the Education of the gifted, v. 32, n. 1, p. 34-53, 2008.

RUDASILL, K. M. et al. Grade and gender differences in gifted students' self-concepts. Journal for the education of the gifted, v. 32, n. 3, p. 340-367, 2009.

SCOTT, J. Gênero: uma categoria útil para análise histórica. Educação e Realidade, Porto Alegre, vol. 20, n. 2, jul./dez 1995, p. 71-99.

UNESCO. Declaração Mundial sobre a Educação para Todos. Plano de ação para satisfazer as necessidades básicas de aprendizagem. Brasília, DF: UNESCO, 1990.

\section{Correspondência}

Mayra Berto Massuda- Universidade Federal de São Carlos, Rod. Washington Luiz. CEP: 13565-905, São Carlos, São Paulo, Brasil.

This work is licensed under a Creative Commons Attribution-NonCommercial 4.0 International (CC BY-NC 4.0) 